\title{
Coherent inelastic backscattering of intense laser light by cold atoms
}

\author{
V. Shatokhin, ${ }^{1,2}$ C. A. Müller, ${ }^{3}$ and A. Buchleitner ${ }^{2}$ \\ ${ }^{1}$ B. I. Stepanov Institute of Physics, National Academy of Sciences of Belarus, Skaryna Ave. 70, BY-220072 Minsk, Belarus \\ ${ }^{2}$ Max-Planck-Institut für Physik komplexer Systeme, \\ Nöthnitzer Str. 38, D-01187 Dresden, Germany \\ ${ }^{3}$ Physikalisches Institut, Universität Bayreuth, D-95440 Bayreuth, Germany
}

(Dated: November 19, 2004)

\begin{abstract}
We present a nonperturbative treatment of coherent backscattering of intense laser light from cold atoms, and predict a nonvanishing backscattering signal even at very large intensities, due to the constructive (self-)interference of inelastically scattered photons.
\end{abstract}

PACS numbers: $42.50 . \mathrm{Ct}, 42.25 . \mathrm{Dd}, 32.80-\mathrm{t}, 42.25 . \mathrm{Hz}$

When a plane wave of arbitrary nature is incident upon a disordered medium of scatterers, the backscattered intensity is an interference pattern of all coherent partial amplitudes containing detailed information on the sample configuration. Under an ensemble average, interference between uncorrelated amplitudes is washed out, except for a small angular range around exact backscattering, where the average intensity may exhibit a narrow peak. This peak results from constructive interference between multiple scattering probability amplitudes counterpropagating along direct and reversed paths [1, 2]. This phenomenon is called Coherent Backscattering (CBS) and was for the first time demonstrated with samples of polystyrene particles [3]. The CBS enhancement factor $\alpha$, the ratio of the total intensity at exact backscattering to the background intensity, measures the coherence of counterpropagating amplitudes responsible for localization effects. Recently, CBS of light has been imported to the quantum realm with clouds of cold atoms [4, 5, 6]. An important leitmotiv of these studies is the robustness of the underlying interference effect with respect to fundamental quantum mechanical dephasing mechanisms, such as spin-flip (of the incoming radiation, which carries a polarization degree of freedom) 7] or inelastic scattering. This has important repercussions for the transition from weak to strong (in Anderson's sense) localization of light in disordered atomic samples [8], and also for potential technological applications such as random lasers [9]. While in the regime of weak, perturbative atom-field coupling, the partial destruction of CBS due to spin-flip like processes - induced by the multiple degeneracy of the atomic transition driven by the incident radiation - has been demonstrated experimentally and analysed theoretically in quite some detail, experiments and theory only now start to probe the strong coupling limit, where inelastic photon-atom scattering processes prevail. First experimental results on Sr (driving the ${ }^{1} S_{0} \rightarrow{ }^{1} P_{1}$ transition with its nondegenerate ground state, hence in the absence of spin-flip) [ 6 ] indeed demonstrate the reduction of the CBS enhancement factor with increasing intensity of the injected field, for values $s=\Omega^{2} / 2\left(\Delta^{2}+\gamma^{2}\right)<1$ of the atomic satura- tion parameter (where $\Omega$ is the Rabi frequency induced by the driving, $\gamma$ half the spontaneous decay rate of the excited atomic level, and $\Delta$ the detuning of the injected laser frequency from the exact atomic resonance). A first scattering theoretical treatment 10] identified the origin of such suppression in the availability of which-path information through inelastically scattered photons: reversed paths can be distinguished by the detection of photons of different frequency. However, this treatment, still perturbative in the field intensity, cannot address the limit of large saturation parameters $s \geq 1$ - with an emerging Mollow triplet [11] in the single atom resonance fluorescence - and, in particular, makes no prediction on the crossover from dominantly elastic to essentially inelastic CBS, nor on the CBS enhancement factor in the deep inelastic limit. In the present Letter, we enter this regime, starting from a general master equation which allows for a nonperturbative treatment of the atom-field coupling. As we will see, even inelastically scattered photons give rise to a nonvanishing CBS signal.

We start out from the elementary toy model of CBS a laser field scattering off two atoms with labels 1 and 2 , placed at a fixed [12] distance $r_{12} \gg \lambda=2 \pi / k_{L}$, with $\mathbf{k}_{L}$ the wave vector of the incident field. It is known from the perturbative treatment of CBS that double scattering (on two atoms) provides the leading contribution to the CBS signal, since this is the lowest order process which gives rise to time reversed scattering amplitudes which can interfere constructively. We expect that this scenario also allows for a qualitative assessment of the nonlinear atomic response in the regime of high laser intensities, whilst propagation effects in the bulk of the scattering medium are certainly beyond reach of this model. Disorder will be mimicked by a suitable average over the atomic positions. Our model also neglects the acceleration of atoms out of resonance, which certainly becomes important at very high intensities, but can be experimentally compensated for by shortening the CBS probe duration, as realized in [ $\underline{6}$. We focus exclusively on the photon coupling to the internal atomic degrees of freedom.

With these premises, the average backscattered in- 


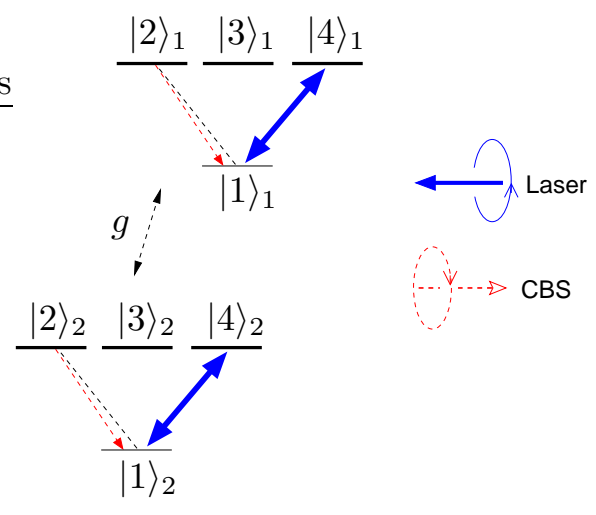

FIG. 1: (Color online) Elementary configuration for coherent backscattering (CBS) of intense light (thick arrows) by two isotropic dipolar transitions in the helicity preserving polarization channel (dashed arrows). The sublevels $|1\rangle$ and $|3\rangle$ of both atoms have magnetic quantum number $m=0$; sublevels $|2\rangle$ and $|4\rangle$ correspond to $m=-1$ and $m=1$, respectively.

tensity can be derived from the correlation functions of the atomic dipoles which emit the detected signal [14, 15]. We specialize to the scenario of the Sr experiments, with a nondegenerate atomic dipole transition $J_{g}=0 \rightarrow J_{e}=1$, driven by laser photons with right circular polarization on the sublevels $|1\rangle \rightarrow|4\rangle$ - see Fig. 1] The scattered light is detected in the helicity preserving channel (i.e., of photons which originate from the $|2\rangle \rightarrow|1\rangle$ transition), where single scattering is absent.
Thus we obtain, up to an irrelevant prefactor, the expectation value for the stationary intensity scattered into the direction $\mathbf{k}$ close to the backward direction $-\mathbf{k}_{L}$,

$$
\langle I\rangle_{\mathrm{ss}}=\left\langle\sigma_{22}^{1}\right\rangle_{\mathrm{ss}}+\left\langle\sigma_{22}^{2}\right\rangle_{\mathrm{ss}}+2 \operatorname{Re}\left(\left\langle\sigma_{21}^{1} \sigma_{12}^{2}\right\rangle_{\mathrm{ss}} e^{i \mathbf{k} \cdot \mathbf{r}_{12}}\right),
$$

where $\sigma_{k l}^{\alpha} \equiv|k\rangle_{\alpha}\left\langle\left. l\right|_{\alpha}\right.$, for atom $\alpha$. The steady-state values for correlation functions of the form $\left\langle\sigma_{i j}^{\alpha}\right\rangle_{\mathrm{ss}}$ or $\left\langle\sigma_{i j}^{\alpha} \sigma_{k l}^{\beta}\right\rangle_{\mathrm{ss}}$ can be found from the master equation [16]

$$
\dot{Q}=\sum_{\alpha=1}^{2} \mathcal{L}_{\alpha} Q+\sum_{\alpha \neq \beta=1}^{2} \mathcal{L}_{\alpha \beta} Q
$$

where the Liouvillians $\mathcal{L}_{\alpha}$ and $\mathcal{L}_{\alpha \beta}$ govern the evolution of an arbitrary atomic operator $Q$ for independent and dipole-dipole interacting (through the exchange of one or several photons) atoms, respectively. $Q$ stands for an operator from the complete set of operators acting on a tensor product of Hilbert spaces of individual atoms. For our choice of the atomic structure shown in Fig. 1 .

$$
Q \in \underbrace{\left\{\sigma_{11}^{1}, \cdots, \sigma_{44}^{1}\right\} \otimes\left\{\sigma_{11}^{2}, \cdots, \sigma_{44}^{2}\right\}}_{256 \text { operators }} .
$$

The explicit form of the interaction-picture Liouvillians $\mathcal{L}_{\alpha}$ and $\mathcal{L}_{\alpha \beta}$ derived in the standard dipole, rotatingwave, and Born-Markov approximations can be shown to read:

$$
\begin{aligned}
\mathcal{L}_{\alpha} Q & =-i \Delta\left[\mathbf{D}_{\alpha}^{\dagger} \cdot \mathbf{D}_{\alpha}, Q\right]-\frac{i}{2}\left[\Omega_{\alpha}\left(\mathbf{D}_{\alpha}^{\dagger} \cdot \varepsilon_{L}\right)+\Omega_{\alpha}^{*}\left(\mathbf{D}_{\alpha} \cdot \varepsilon_{L}^{*}\right), Q\right]+\gamma\left(\mathbf{D}_{\alpha}^{\dagger} \cdot\left[Q, \mathbf{D}_{\alpha}\right]+\left[\mathbf{D}_{\alpha}^{\dagger}, Q\right] \cdot \mathbf{D}_{\alpha}\right) \\
\mathcal{L}_{\alpha \beta} Q & =\mathbf{D}_{\alpha}^{\dagger} \cdot \overleftrightarrow{\mathbf{T}}(g, \hat{\mathbf{n}}) \cdot\left[Q, \mathbf{D}_{\beta}\right]+\left[\mathbf{D}_{\beta}^{\dagger}, Q\right] \cdot \overleftrightarrow{\mathbf{T}}^{*}(g, \hat{\mathbf{n}}) \cdot \mathbf{D}_{\alpha}
\end{aligned}
$$

where $\Delta=\omega_{L}-\omega_{0}$ is the detuning, $\Omega_{\alpha}=\Omega e^{i \mathbf{k}_{L} \cdot \mathbf{r}_{\alpha}}$ is the atomic (coordinate-dependent) Rabi frequency, and $\varepsilon_{L}$ fixes the polarization of the laser field.

$$
\mathbf{D}_{\alpha}=-\varepsilon_{-1} \sigma_{12}^{\alpha}+\varepsilon_{0} \sigma_{13}^{\alpha}-\varepsilon_{+1} \sigma_{14}^{\alpha}
$$

is the lowering dipole operator of atom $\alpha$, with $\varepsilon_{ \pm 1}, \varepsilon_{0}$ the unit vectors of the spherical basis. The radiative dipole-dipole interaction due to exchange of photons between the atoms is described by the tensor $\overleftrightarrow{\mathbf{T}}(g, \hat{\mathbf{n}})=$ $\gamma g \overleftrightarrow{\boldsymbol{\Delta}}$, where $\overleftrightarrow{\boldsymbol{\Delta}}=\overleftrightarrow{\mathbb{1}}-\hat{\mathbf{n}} \hat{\mathbf{n}}$ is the projector on the plane defined by the vector $\hat{\mathbf{n}}$ connecting atom 1 and 2 , and

$$
g=i 3 \frac{e^{i k_{0} r_{12}}}{2 k_{0} r_{12}}
$$

where $k_{0}=\omega_{0} / c$, is the small coupling constant $|g| \ll 1$ in the far-field limit $k_{0} r_{12} \gg 1$, where we neglect nearfield interaction terms of order $1 /\left(k_{0} r_{12}\right)^{2}$ and $1 /\left(k_{0} r_{12}\right)^{3}$ (which, at higher atomic densities, could also be retained in our formalism).

Transforming the operator equation (2) to a system of 255 linear coupled differential equations for the atomic correlation functions, we can solve for the physical quantities which enter the expression (11) for the detected intensity. In doing so, we furthermore take advantage of the far field limit $k_{0} r_{12} \gg 1$, and expand the correlation functions up to second order, $\langle(\ldots)\rangle_{\mathrm{ss}}^{[2]}$, in the dipole-dipole coupling constant (7). The double scattering contribution to the CBS signal, detected in the helicity preserving channel, is then precisely given by terms proportional to $g^{2}$, since it stems from the exchange of two photons between the atoms, along a 'direct' and its 'reversed' path. Finally, the CBS signal is obtained after an elementary configuration average $\langle\ldots\rangle_{\text {conf. }}$ defined through the following twofold procedure: (i) isotropic averaging of the 


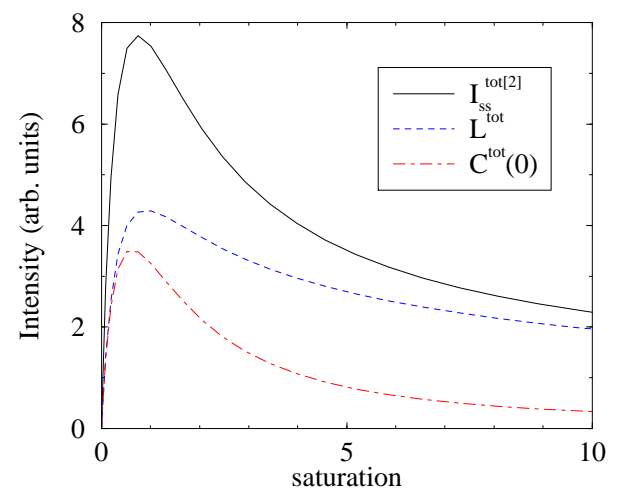

FIG. 2: (Color online) Total intensities of the ladder, the crossed terms, and their sum $I_{\mathrm{ss}}^{\text {tot }}{ }^{[2]}$, in the helicity preserving channel, as functions of the saturation parameter $s$ at resonance, $\Delta=0$.

relative orientation $\hat{\mathbf{r}}_{12}$ over the unit sphere; (ii) uniform averaging of the distance $r_{12}$ over an interval of the order of $\lambda$, around a mean value given by the mean free path. After this simple procedure all terms relevant for the calculation of the CBS enhancement factor survive, whereas all the irrelevant terms vanish.

We thus arrive at our final expression for the total second-order intensity

$$
I_{\mathrm{ss}}^{\mathrm{tot}[2]}(\theta)=L^{\mathrm{tot}}+C^{\mathrm{tot}}(\theta),
$$

a sum of the total ladder (or background), $L^{\text {tot }}$, and total crossed (or interference) term $C^{\text {tot }}(\theta)$, with $\theta$ the observation angle of the scattered intensity with respect to the backward direction. In terms of the second order atomic correlation functions, $L^{\text {tot }}$ and $C^{\text {tot }}(\theta)$ are given by

$$
\begin{aligned}
L^{\text {tot }} & =\left\langle\left\langle\sigma_{22}^{1}\right\rangle_{\mathrm{ss}}^{[2]}+\left\langle\sigma_{22}^{2}\right\rangle_{\mathrm{ss}}^{[2]}\right\rangle_{\text {conf. }} \\
C^{\text {tot }}(\theta) & =2 \operatorname{Re}\left\langle\left\langle\sigma_{21}^{1} \sigma_{12}^{2}\right\rangle_{\mathrm{ss}}^{[2]} e^{i \mathbf{k} \cdot \mathbf{r}_{12}}\right\rangle_{\text {conf. }}
\end{aligned}
$$

Therefrom we deduce the main quantifier of CBS, the enhancement factor

$$
\alpha=\frac{L^{\mathrm{tot}}+C^{\mathrm{tot}}(0)}{L^{\mathrm{tot}}} .
$$

Figure 2 shows our results for the total CBS intensity as well as its components $L^{\text {tot }}$ and $C^{\text {tot }}(0)$, as a function of the saturation parameter, at exact resonance $(\Delta=0)$. The behavior of $I_{\mathrm{ss}}^{\mathrm{tot}[2]}$ shows that the double scattering intensity behaves markedly different from that of an isolated atom. Whilst the scattering intensity from an isolated atom $I^{[0]} \propto s /(1+s)$ is known to saturate for large $s$ [15], the double scattering intensity exhibits a maximum at $s \simeq 0.7$, followed by gradual decrease $\sim s^{-1}$ for large $s$ : At high laser intensities, more and more photons are scattered inelastically, and are therefore less likely to undergo resonant interaction with the second atom.

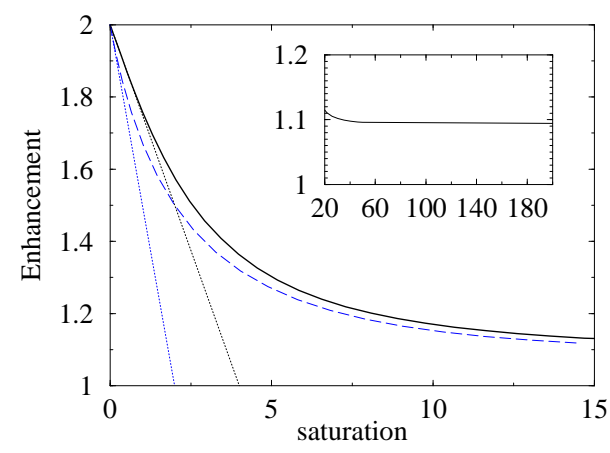

FIG. 3: (Color online) Enhancement factor $\alpha$ in the helicity preserving channel, versus saturation parameter $s$. Solid curve: on resonance $(\Delta=0)$, dashed curve: off resonance $(\Delta=\gamma)$. Straight lines represent the perturbative prediction $2-(1+\delta) s / 4$ of [10]. Inset: The finite enhancement $\alpha_{\infty} \simeq 1.09$ signals residual photon (self-)interference, even in the deep inelastic regime.

The enhancement factor $\alpha(s)$ follows directly from the above quantities. As shown in Fig. 3] it decays monotonously from its weak field limit $\alpha(0)=2$. In qualitative agreement with the experiment [a] , this decay is faster for finite detuning $\Delta=\gamma$. For small values of $s, \alpha$ is well approximated by the linear decay $2-(1+\delta) s / 4$, with $\delta=(\Delta / \gamma)^{2}$, derived within the scattering picture [10]. For large values of $s$ (inset), however, $\alpha$ saturates at a value $\alpha_{\infty} \simeq 1.09$ strictly larger than unity, whilst one would expect vanishing contrast (i.e., $\alpha=1$ ) for scattering from two independent atoms [17. Hence, the (self-)interference of inelastically scattered photons unambiguously contributes to the crossed term $C^{\text {tot }}(0)$. Note that this observation bears some similarity to CBS with photons from degenerate Raman transitions, which were shown to yield an important contribution to the CBS contrast, even in the limit of infinite ground state degeneracy [13], as well as to the residual CBS enhancement in optically active media at high magnetic fields [18].

In contrast, elastically scattered photons remain perfectly coherent, and contribute to the CBS intensity with a constrast two, for any $s$. To see this, we just need to extract the purely elastic component of the signal from the total yield in eq. (11). Since the detected intensity $\langle I\rangle_{\mathrm{ss}}$ is nothing but the autocorrelation function of the source field amplitudes radiated by the atomic dipoles, its elastic part $\langle I\rangle_{\mathrm{ss}}^{\mathrm{el}}$ is generated by the classical dipoles induced by the injected radiation - this is by their average, nonfluctuating parts $\left\langle\sigma_{i \neq j}^{\alpha}\right\rangle_{\mathrm{ss}}[15]$. Hence, $\langle I\rangle_{\mathrm{ss}}^{\mathrm{el}}$ is given by the product of the expectation values of the atomic dipoles:

$$
\langle I\rangle_{\mathrm{ss}}^{\mathrm{el}}=\left|\left\langle\sigma_{21}^{1}\right\rangle_{\mathrm{ss}}\right|^{2}+\left|\left\langle\sigma_{21}^{2}\right\rangle_{\mathrm{ss}}\right|^{2}+2 \operatorname{Re}\left(\left\langle\sigma_{21}^{1}\right\rangle_{\mathrm{ss}}\left\langle\sigma_{12}^{2}\right\rangle_{\mathrm{ss}} e^{i \mathbf{k} \cdot \mathbf{r}_{12}}\right) .
$$

A power series expansion of the right hand side of (12) 
to second order in the coupling $g$ leaves only symmetrically factorized combinations of the form $\left\langle\sigma_{21}^{\alpha}\right\rangle^{[1]}\left\langle\sigma_{12}^{\beta}\right\rangle^{[1]}$. Asymmetric combinations, like $\left\langle\sigma_{21}^{\alpha}\right\rangle^{[2]}\left\langle\sigma_{12}^{\beta}\right\rangle^{[0]}$, do not contribute to the signal since the $|1\rangle \leftrightarrow|2\rangle$ transitions are not laser-driven (see Fig. 1), hence $\left\langle\sigma_{12}^{\beta}\right\rangle^{[0]}$ vanishes. Evaluation of the correlation functions by symbolic calculus, together with the configuration average described above, finally provides an analytic expression for the elastic ladder and crossed terms:

$$
L^{\mathrm{el}}=C^{\mathrm{el}}(0)=24 \pi|g|^{2} \frac{1}{1+\delta} \frac{s}{(1+s)^{4}} .
$$

Expression (13) shows that the elastic ladder and crossed terms are equal for any $s$, as to be expected from reciprocity arguments 13. These elastic components decay like $s^{-3}$ at large saturation, much faster than the total intensities that decrease like $s^{-1}$ (cf. Fig. 2). This proves that the residual CBS enhancement $\alpha_{\infty}$ is entirely due to the (self-)interference of inelastically scattered photons. Furthermore, expression (13) shows that the elastic part of the double scattering intensity exhibits a maximum at $s=1 / 3$, slightly below the departure of $\alpha(s)$ from the perturbative prediction of [10] in Fig. [3] Consistently, an expansion of (13) to second order in $s$ reproduces the expression $L^{\mathrm{el}}=C^{\mathrm{el}}(0) \sim s-4 s^{2}$ derived in [10]. Note that the crossover to the nonlinear regime for double scattering occurs at a value of $s$ three times smaller than for an isolated atom, where $I^{\mathrm{el}[0]} \propto s /(1+s)^{2}$ exhibits a maximum at $s=1$. This has a transparent interpretation, by virtue of factorizing eq. (13) into (i) the elastic intensity $I^{\mathrm{el}[0]}$ scattered by the first strongly driven atom, (ii) the total scattering cross section $\sigma^{\text {tot }} \propto 1 /(1+\delta)(1+s)$ of the second atom, and (iii) the relative weight $I^{\mathrm{el}[0]} / I^{\text {tot }[0]}=\sigma^{\mathrm{el}} / \sigma^{\text {tot }}=$ $\left(\gamma^{2}+\Delta^{2}\right) /\left(\gamma^{2}+\Omega^{2} / 2+\Delta^{2}\right)=1 /(1+s)$ 15] of elastic processes therein. Obviously, higher order scattering processes than considered in our present contribution must unavoidably push the crossover value of $s$ to even smaller values.

In conclusion, we have presented the first study of coherent backscattering of intense laser light from saturated dipole transitions. The CBS enhancement decreases monotonously as a function of $s$, but, remarkably, coherence is - partially - preserved in the deep inelastic limit of the two-atom response to intense laser radiation, since also inelastically scattered photons can interfere with themselves, along time-reversed paths. Consequently, CBS should also have an imprint on the spectrum of the scattered radiation, as well as on its photocount statistics, which are both directly accessible in the framework of our present approach, as well as in laboratory experiments. Furthermore, let us note that our present results are also relevant in the somewhat different context of Young's double slit experiments with two atoms [17. In contrast to the forward Young-type interference that necessarily decoheres for $s \rightarrow \infty$, since the photon visits two different, uncoupled atoms, a backscattering experiment, with appropriate polarization sensitive excitation and detection, must lead to a finite interference contrast.

We would like to thank Dominique Delande, Benoît Grémaud, Christian Miniatura, Mikhail Titov, and Thomas Wellens for stimulating discussions.

[1] E. Akkermans, G. Montambaux, J.-L. Pichard, and J. Zinn-Justin (Eds.) Mesoscopic Quantum Physics (Elsevier, Amsterdam, 1994).

[2] P. Sheng, Introduction to Wave Scattering, Localization and Mesoscopic Phenomena (Academic Press, San Diego, 1995).

[3] Y. Kuga and A. Ishimaru, J. Opt. Soc. Am. A 1, 831 (1984); M. P. van Albada and A. Lagendijk, Phys. Rev. Lett. 55, 2692 (1985); P. E. Wolf and G. Maret, ibid. 2996.

[4] G. Labeyrie, F. de Tomasi, J.-C. Bernard, C. A. Müller, C. Miniatura and R. Kaiser, Phys. Rev. Lett. 83, 5266 (1999).

[5] P. Katalunga, C. I. Sukenik, S. Balik, M. D. Havey, D. V. Kupriyanov, and I. M. Sokolov, Phys. Rev. A 68, 033816 (2003).

[6] T. Chanelière, D. Wilkowski, Y. Bidel, R. Kaiser, and C. Miniatura, Phys. Rev. E 70, 036602 (2004).

[7] C. A. Müller and C. Miniatura, J.Phys. A 35, 10163 (2002).

[8] Th. M. Nieuwenhuizen, A. L. Burin, Yu. Kagan, and G. V. Shlyapnikov, Phys. Lett. 184A, 360 (1994).

[9] H. Cao, Y. Ling, J. Y. Xu, C. Q. Cao, and Prem Kumar, Phys. Rev. Lett. 86, 4524 (2001).

[10] T. Wellens, B. Gremaud, D. Delande, and C. Miniatura, Phys. Rev. A 70, 023817 (2004).

[11] B. R. Mollow, Phys. Rev. 188, 1969 (1969).

[12] At typical temperatures of the cloud of about a few $\mathrm{mK}$ [4], the Doppler shift associated with the thermal motion is negligible, yet the kinetic energy of an atom is much larger than the recoil energy, thus allowing a classical description of the atomic position [13].

[13] C. A. Müller, T. Jonckheere, C. Miniatura, and D. Delande, Phys. Rev. A 64, 053804 (2001).

[14] G. S. Agarwal, Quantum Statistical Theories of Spontaneous Emission and their Relation to Other Approaches (Springer-Verlag, Berlin, 1974).

[15] C. Cohen-Tannoudji, J. Dupont-Roc, G. Grynberg, Atom-Photon Interactions (John Wiley \& Sons Inc, New York, 1992).

[16] R. H. Lehmberg, Phys. Rev. A 2, 883 (1970); D. F. V. James, ibid. 47, 1336 (1993); J. Guo and J. Cooper, ibid. 51, 3128 (1995).

[17] P. Kochan, H. J. Carmichael, P. R. Morrow, and M. G. Raizen, Phys. Rev. Lett. 75, 45 (1995).

[18] A. S. Martinez and R. Maynard, Phys. Rev. B 50, 3714 (1994). 